\title{
APPLICATION OF PORTABLE FORCE PLATFORMS EQUIPPED WITH A DEVICE FOR MEASURING POSITION AND ORIENTATION
}

\author{
Patrik Kutílek $^{a, *}$, Slávka VíteČKová ${ }^{a}$, ZdenĚK Svoboda $^{b}$, Jan HejdA ${ }^{a}$ \\ ${ }^{a}$ Faculty of Biomedical Engineering, Czech Technical University in Prague, nam. Sitna 3105, 27201 Kladno, the \\ Czech Republic \\ ${ }^{b}$ Faculty of Physical Culture, Palacky University of Olomouc, Krizkovskeho 8, 77147 Olomouc, the Czech \\ Republic \\ * corresponding author: kutilek@fbmi.cvut.cz
}

\begin{abstract}
With the development of modern technology for studying the movements of the human body, a large number of new measurement techniques are being applied. However, a complex and expensive device is not always the best one for personal requirements or for professional needs. We have designed and tested a new universal way to study the forces under the feet based on force platforms equipped with a device for measuring position and orientation. The device for measuring position and orientation is used to identify the relative positions of the two inexpensive force platforms in 3-D space. Knowledge of the relative positions and orientations of the force platforms allows us to adjust the two force platforms in 3-D space. The technique allows the force platform system for measuring and calculating the forces under the feet and the position of the center of pressure to be set up rapidly. The new technique, based on inexpensive portable force platforms equipped with a device for measuring position and orientation, is used and described for the first time here. This work attempts to describe potential ways of applying the technique.
\end{abstract}

KEYWORDS: portable force platform, center of pressure, gyroscope, MoCap system.

\section{INTRODUCTION}

In medical practice, there is no appropriate widelyused system based on inexpensive portable force platforms equipped with a device for measuring and calculating the forces under the feet and the position of the center of pressure $(\mathrm{CoP})$. Research and clinical studies of gait or postural stability are often based only on expensive non-portable force plates. There are no widely used and appropriate techniques for measuring stability in various situations [1, 2].

At the present time, posturography is the most widely-used method for assessing postural stability [3]. Static or dynamic posturography is mainly used in research, sometimes in conjunction with other devices for analysing body movement activity. This technique uses force platforms to measure the forces exerted by the subject's feet [4. Posturography assesses a patient's balance, i.e. postural stability, by examining the trajectory of the CoP [5, 6]. We can use a single force platform, on which the subject stands with his feet placed in a fixed position. Two separate platforms can also be used, one under each foot, which allows the forces exerted by each foot to be recorded separately [3. With these last-mentioned devices, the position of the feet is not fixed and can be variable. The most widely-studied CoP parameters are trajectory length, maximum amplitude of the displacement of $\mathrm{CoP}$, etc. Kistler and ATMI are examples of a commercial platform. Knowledge about the distances between the force sensors, i.e. separate platforms for measuring the forces, is important for calculating the CoP position. The values for the distances between the force sensors are used for calculating the $\mathrm{CoP}$ position by equations of static equilibrium of forces and torques [7. Pressure distribution platforms (Novel Emed, RS-Scan Footscan, etc.) are also used for studying the CoP position. The platforms are connected to the data acquisition module. The data acquisition module collects the output from the platforms and sends the data to a PC. Based on the pressure distribution and equations of the static equilibrium of forces and torques, special software calculates the trajectory length, the area enclosed by the trajectory, the average speed of CoP along its path, etc.

The disadvantage of current platform systems is that they are designed to measure the position of the $\mathrm{CoP}$ during complex movements of patients only if the platform systems are large (e.g. plantar pressure measuring plate) or geometrically complex (e.g. in-shoe insoles that measure pressure), or the systems have predefined positions and orientations of the platforms in $3 \mathrm{D}$ space. These expensive pressure measuring plates or in-shoe insoles (RSscan International, Dynafoot or Footscan) monitor the pressure distribution under the feet or shoes during walking. A problem with these devices is that they identify the CoP position inaccurately, or not at all. For this reason, parallel measurement of $\mathrm{CoP}$ under both feet using 


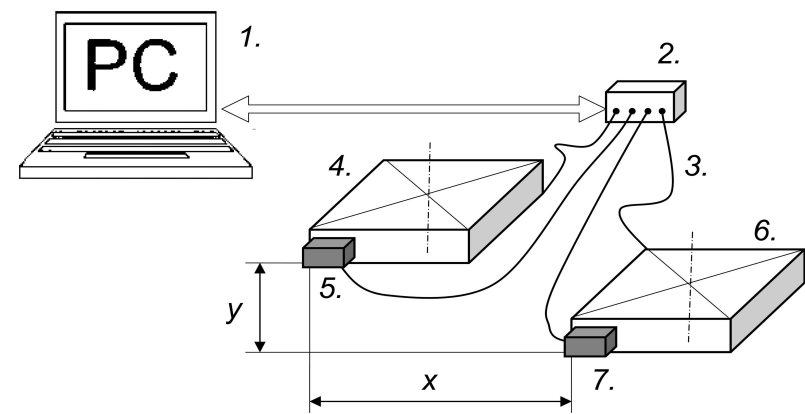

FiguRE 1. Diagram of the designed system with two sensors of the relative positions/orientations of portable platforms; 1) module for processing the measured data, and a display to view information on the position and size of the resultant contact forces; 2) data acquisition module for collecting data from the platforms and tracking sensors; 3) data cables; 4) and 6 ) platforms for detecting the position and size of the resultant contact forces under the feet; 5) and 7) sensors mounted on platforms to detect their relative positions and orientation in 3D space.

two force platforms (i.e. one foot on one platform) is introduced. The pre-defined fixed geometry of the relative positions/orientations of the two platforms for identifying the CoP position is used in systems such as Tetrax, manufactured by Sunlight Medical Ltd. A solution using more than one platform for studying the CoP position is also used in systems such as Cyber-Sabots. In this case, two platforms are used. The platforms are always situated a fixed distance apart, and they can rotate against each other. Two platforms situated a fixed distance apart are also always used with other systems, such as MoCap systems for studying the movement of body segments. More than two platforms situated a fixed distance apart have also been used for studying the CoP position during the transition from sitting to standing $[8]$. However, these systems are currently expensive, they are not universal and quickly adjustable, or they are patent-protected. Hence, the CoP position during the transition from sitting to standing, or while walking up and down steps, cannot be studied with widelyused platforms. The aim of this paper is to show a system using inexpensive force or pressure platforms for measuring the relative position and orientation of the platforms in $3 \mathrm{D}$ space.

\section{Methods}

\subsection{Measurement Devices}

The disadvantages of current systems for studying the CoP position are eliminated by using a new method for identifying the relative positions/orientation of two or more platforms. This method allows us quickly and easily to set up platforms for measurements of a new type. We used two Wii Fit Balance Boards (from Nintendo Ltd.) in the design of our system for studying the CoP position. Wii Balance Boards is a force platform. The platform uses Bluetooth technology and contains four force sensors that are used to measure the user's CoP. The platforms are connected to a PC or laptop using Bluetooth.

The proposed method is based on attaching one or more 3D position/orientation sensors to the platforms (Fig. 1), thereby enabling the relative positions of the platforms in 3D space to be detected. The sensor can generally be attached on the basis of various principles for detecting the position and orientation to the platforms. In our case, we used gyro-accelerometer sensors. The gyro-accelerometer sensor works on the principle of sensing the angles of rotation in three directions and the acceleration in three directions in 3D space. Using information about the angles and accelerations when moving the gyro-accelerometer sensor, the computational algorithms accurately determine the instantaneous orientation of the sensor. They also, less accurately, determine the instantaneous position of the sensor in $3 \mathrm{D}$ space. When there is more than one gyro-accelerometer, the relative position and orientation of the gyro-accelerometers can be evaluated mathematically by algorithms. Example of these systems are Xsens, Trivisio Colibri, etc.

Another way to detect the relative positions of the two boards is by using a six-degree-of-freedom electromagnetic tracking sensor device. The device accurately computes the position and orientation of a tiny receiver attached to the platform as it moves through space. This sensor can inform us about the position $(x$, $y$, and $z$ Cartesian coordinates) and orientation (azimuth, elevation and roll) of the platform. Examples of these systems are Polhemus Fastrak, Liberty, etc. The lowest price for systems using the electromagnetic principle is approximately EUR 5000. Alternatively, the relative positions of the platforms can be detected with the use of acoustic systems, where ultrasonic source(s) and ultrasonic receiving module(s) are used for position sensing. Ultrasonic sources or receivers can be attached to the platforms. Examples of these systems are InterSense 600, IS 900, etc. The last option for recording the position and orientation of the platforms in 3D space is the use of optical systems. Data acquisition is traditionally implemented using special markers attached to an object. The object can be a platform. These systems can provide data with 3 degrees of freedom for each marker on the platform, and rotational information must be inferred from the relative orientation of three or more markers on one platform. We can use an optical system with passive or active markers. A passive optical system uses markers coated with a retroreflective material to reflect light that is generated near the camera lens. In the case of active optical systems, the markers themselves are powered to emit their own light. The transmitted or reflected signal is processed in the camera system and PC, and the position of the markers on the platforms is determined. Passive optical systems include Lukotronic, Optotrak Certus, etc. Active opti- 


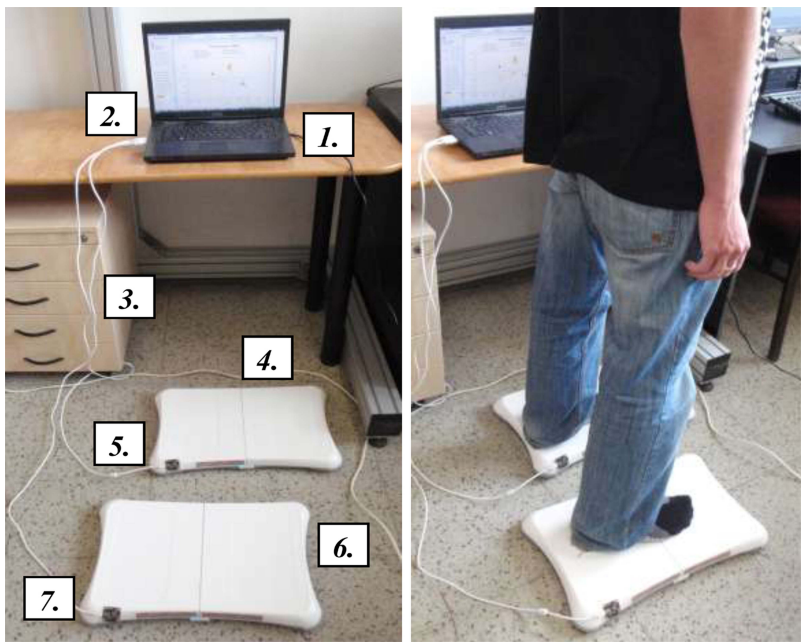

Figure 2. Example of the designed system with two sensors of the relative positions/orientations of portable platforms: 1) the module for processing the measured data and the display for viewing information about the position and size of the resultant contact forces (part of PC); 2) data acquisition module for collecting data (part of PC); 3) data cables; 4) and 6) platforms for detecting the position and size of the resultant contact forces; 5) and 7) sensors mounted on platforms to detect their relative positions and orientation in 3D space.

cal systems include Vicon, Qualisys, etc. This enables the markers to be placed permanently on platforms in laboratories using a camera system of this type. The position of the platforms can be automatically tracked when moved. The platforms are usually fixed and stationary in laboratories, though the optical system can be used to track the position of the platforms during clinical examinations. The lowest price for optical systems is approximately EUR 2500. For all proposed methods, MoCap systems monitor the movement of the platforms, and the processed signal from specific systems is used to identify the instantaneous position and orientation of a platform in $3 \mathrm{D}$ space. This processing can produce information on the relative position and the relative orientation of the platforms in space.

The sensor for measuring the position of the platform is firmly attached and precisely/accurately positioned in a defined point on each platform. The geometric/dimensional parameters, including the size of the platform and the position of the sensor on the platform within the coordinate system of the platform are input information for the calculation algorithm to determine the position/orientation of the platform in 3D space. In our case, we used a Phidgets USB 9DoF position and orientation sensor, composed of an accelerometer, a gyroscope and a magnetometer. The relative positions of the two platforms can be detected using only one or two position sensors. If the position sensor is used on only one platform, only the one platform with the sensor can be moved. The position of the platform without a sensor is constant and pre-defined at the beginning of the measurement. If the sensors are placed on more than one platform, we can measure the mutual position and orientation of the platforms when they move, see Fig. 2. The computational algorithm evaluates the location of all the platforms with sensors in 3D space. The instantaneous CoP position is determined using information about the dimensional parameters of the platforms, the forces measured by the force sensors of the force platforms and the parameters of the mutual position and the orientation of platforms. The CoP position is determined for the body of a patient/subject moving on more than one platform. With at least two force platforms, the $\mathrm{CoP}$ under the soles and also the overall $\mathrm{CoP}$ under both feet, where each foot is placed on only one platform in $3 \mathrm{D}$ space, can be evaluated simultaneously. To determine the relative positions/orientation of the platforms, calibration and location of the platforms in relation to the initial positions in 3D space must be carried out at the beginning of each measurement. After placing the platforms in the initial positions, information about the default positions/orientations is introduced into the calculation algorithm. The platform can be moved arbitrarily in $3 \mathrm{D}$ space after we have defined the initial position of the one platform where the sensor is attached to the only one platform. If sensors are mounted on both platforms, the platforms are arbitrarily placed in space according to the measurement needs, i.e. the methods of clinical examination. After positioning the platform to the desired position/orientation in $3 \mathrm{D}$ space, the position of the CoP can be measured. The computational algorithm processes the measured data and information about the relative position and orientation of the platforms, the geometric dimensions of the platforms and the forces measured by the force sensors of the force platforms.

\subsection{Calculation of CoP POsition}

It is simple to calculate the magnitude of the force under the sole, i.e. the force transferred onto one limb. It is a simple calculation, represented by the sum of the forces on the force sensors of the one platform, i.e. for a sole on board A, Fig.3:

$$
F_{z \mathrm{VA}}=F_{z 1 \mathrm{~A}}+F_{z 2 \mathrm{~A}}+F_{z 3 \mathrm{~A}}+F_{z 4 \mathrm{~A}}
$$

where $F_{z 1 \mathrm{~A}}, \ldots, F_{z 4 \mathrm{~A}}$ are the forces measured by the force sensors of the platform. The moment of the forces in the individual sensors relative to the centre of board OA will be used to identify the point of application of the resultant contact force under the sole representing the point of the $\mathrm{CoP}$ position on one platform:

$$
\begin{aligned}
& M_{x \mathrm{~A}}=p_{y \mathrm{~A}} F_{z \mathrm{VA}} \\
& =a_{\mathrm{A}}\left(F_{z 1 \mathrm{~A}}-F_{z 2 \mathrm{~A}}-F_{z 3 \mathrm{~A}}+F_{z 4 \mathrm{~A}}\right) \\
& \begin{array}{r}
M_{y \mathrm{~A}}=p_{x \mathrm{~A}} F_{z \mathrm{VA}} \\
=b_{\mathrm{A}}\left(F_{z 1 \mathrm{~A}}-F_{z 2 \mathrm{~A}}-F_{z 3 \mathrm{~A}}+F_{z 4 \mathrm{~A}}\right)
\end{array}
\end{aligned}
$$




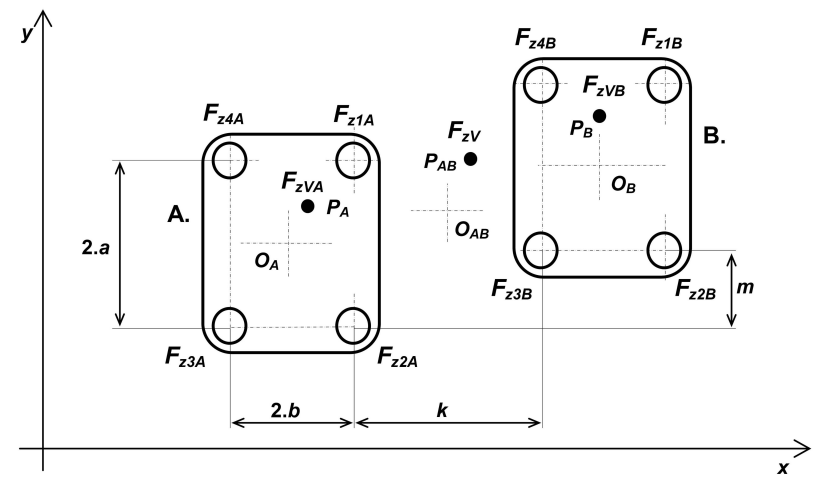

Figure 3. A diagram of two force platforms for contact force measurement under both soles.

where $a_{\mathrm{A}}$ and $b_{\mathrm{A}}$ are dimensions of the platform, i.e. distances of the force sensors. The moments of the forces measured by the four sensors must correspond to the moments generated by force $F_{z \mathrm{VA}}$ acting at point $P_{\mathrm{A}}$ relative to point $O_{\mathrm{A}}$. The solution of the above equations yields $M_{x \mathrm{~A}}, M_{y \mathrm{~A}}$ and by means of these moments we find the point of application of the contact force (i.e. $p_{x} \mathrm{~A}, p_{y \mathrm{~A}}$ ) on the platform $\mathrm{A}$. The point of application of the resultant contact force (i.e. CoP position) for the second platform B will be detected in an identical way.

If we want to detect the $\mathrm{CoP}$ position from two platforms (Fig. 3), we must again first identify the magnitude of the resultant force acting on the two platforms:

$$
\begin{aligned}
F_{z \mathrm{~V}}=F_{z 1 \mathrm{~A}}+ & F_{z 2 \mathrm{~A}}+F_{z 3 \mathrm{~A}}+F_{z 4 \mathrm{~A}} \\
& +F_{z 1 \mathrm{~B}}+F_{z 2 \mathrm{~B}}+F_{z 3 \mathrm{~B}}+F_{z 4 \mathrm{~B}}
\end{aligned}
$$

To be able to identify the point of application of the resultant contact force, let us express the moment of the forces measured by the sensors relative to point $O_{\mathrm{AB}}$, which is situated in the centre of the distance between the platforms. In doing this, we assume identical dimensions, $a=a_{\mathrm{A}}=a_{\mathrm{B}}$ and $b=b_{\mathrm{A}}=b_{\mathrm{B}}$, and parallelism (ensured by gyro sensors) of the two platforms, and their arrangement according to Fig. 3.

$$
\begin{aligned}
& M_{x \mathrm{AB}}=p_{y \mathrm{AB}} F_{z \mathrm{~V}} \\
& =\left(a+\frac{m}{2}\right)\left(F_{z 1 \mathrm{~B}}+F_{z 4 \mathrm{~B}}-F_{z 2 \mathrm{~A}}-F_{z 3 \mathrm{~A}}\right) \\
& \quad+\left(a-\frac{m}{2}\right)\left(F_{z 1 \mathrm{~A}}+F_{z 4 \mathrm{~A}}-F_{z 2 \mathrm{~B}}-F_{z 3 \mathrm{~B}}\right) \\
& M_{y \mathrm{AB}}=p_{x \mathrm{AB}} F_{z \mathrm{~V}} \\
& =\left(\frac{k}{2}+2 b\right)\left(F_{z 3 \mathrm{~A}}+F_{z 4 \mathrm{~A}}-F_{z 1 \mathrm{~B}}-F_{z 2 \mathrm{~B}}\right) \\
& \quad+\frac{k}{2}\left(F_{z 1 \mathrm{~A}}+F_{z 4 \mathrm{~A}}-F_{z 3 \mathrm{~B}}-F_{z 4 \mathrm{~B}}\right) .
\end{aligned}
$$

The moments of the forces measured by the eight sensors must correspond to the moments generated by the resultant force $F_{z \mathrm{~V}}$ acting at point $P_{\mathrm{AB}}$ relative to point $O_{\mathrm{AB}}$. The solution of these equations yields $M_{x \mathrm{AB}}, M_{y \mathrm{AB}}$ and by means of these moments we find

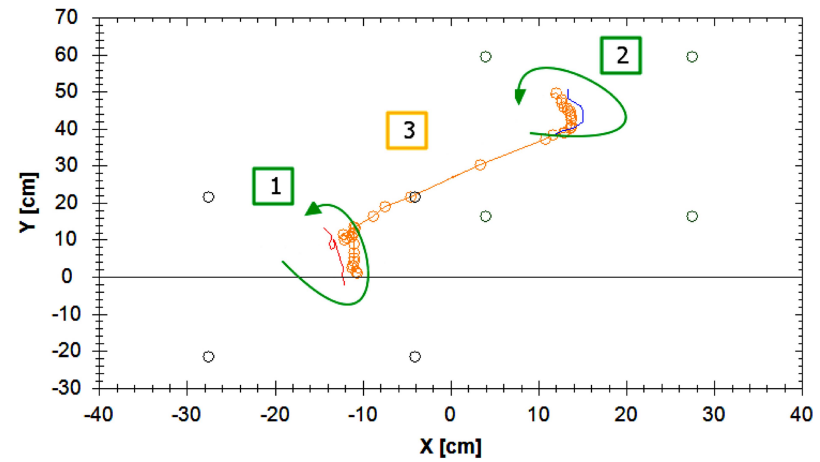

Figure 4. Example of the measured CoP trajectories when walking upstairs; 1) CoP trajectory under the left foot measured by the left platform and, outlined, the main direction of the CoP on the left platform; 2) CoP trajectory under the right foot measured by the right platform and, outlined, the main direction of $\mathrm{CoP}$ on the right platform; 3) calculated trajectory of the total CoP.

the point of application $\left(p_{x \mathrm{AB}}, p_{y \mathrm{AB}}\right)$ of the resultant contact force. This point corresponds to the $\mathrm{CoP}$ position of the whole body. If we use more platforms or their mutual rotation, the above formulas can be used or modified in the calculation algorithm.

\section{EXPERIMENTAL VERIFICATION}

The proposed system based on two platforms was tested and used for monitoring the total center of pressure (CoP) of the body. The system was tested on healthy subjects (students of the Czech Technical University). The proposed system and software allow us not only to evaluate CoP, but also to analyze the signal frequency; the sampling frequency of the system/two platforms is $64 \mathrm{~Hz}$. As described above, the technique allows CoP to be measured for more complex movements and positions, such as the posture of the body. Testing was performed using normal walking, walking upstairs, and the transition from sitting to standing. In order to study the gait of the subjects, the right platform was located $38 \mathrm{~cm}$ from the initial (original) position, i.e. the left platform, in the direction of walking. The individual steps are $25 \mathrm{~cm}$ high. The proposed set allows us easily to measure and identify the position of the $\mathrm{CoP}$ on individual platforms and the total CoP under both feet by using two force platforms, see Fig. 4

We will use an example of recorded data (measurements for one subject) to describe the measurement results and the potential of the proposed system and software. The circular marks on the trajectory of $\mathrm{CoP}$, Fig. 4. correspond to the moments of the measurement, i.e. the recording (sampling frequency: $64 \mathrm{~Hz}$ ). We can therefore read directly from the graph (Fig. 4) that the subject changed CoP position quickly or slowly. From the distance of the circular marks representing moments of the instantaneous total CoP position, we see from the great density of the markers that there 


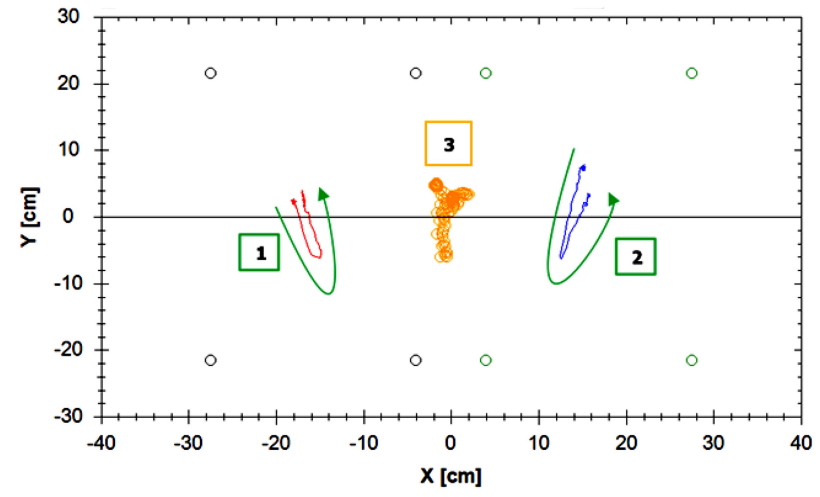

Figure 5. Example of the measured CoP trajectories during the transition from sitting to standing; 1) CoP trajectory under the left foot measured by the left platform and, outlined, the main direction of $\mathrm{CoP}$ on the left platform; 2) CoP trajectory under the right foot measured by the right platform and, outlined, the main direction of CoP on the right platform; 3 ) calculated trajectory of the total CoP.

was a slower movement of the feet on each platform. When the left foot first contacts the platform, we can see that the total $\mathrm{CoP}$ initially moves from the back to the front position, with no major deviations to the left or right. After the second leg contacts the right platform, the total CoP moves forward and the left leg is subsequently lifted from the lower left platform. The trajectory of the total CoP therefore corresponds to the $\mathrm{CoP}$ trajectory under the left and right foot measured by the left and right platform. CoP moves from the back position to the front position, but the path is directed slightly to the left. The slight difference between the CoP trajectories identified by the individual platforms and the $\mathrm{CoP}$ trajectory identified by the computational algorithms and parallel measurement by two platforms is due to small inaccuracies in the detection of the relative positions and orientation of the two platforms in 3D space.

An example of other uses of the system is for studying the CoP trajectory during the transition from sitting to standing, Fig. 5. The system was tested by assuming the initial position, i.e. both platforms are placed side-by-side. First, the subject sat on a standard office chair, and then the subject stood on the platforms in an upright standing position when instructed by a physician. Diagrams of the CoP trajectories of the feet and the total CoP trajectory of the body indicate that the total $\mathrm{CoP}$ was initially in the front part of the platforms, then it moved to the back part of the platforms (under both heels), and then it again moved to the front part of the platforms. It is also obvious that in this example the left parts of the feet are more loaded, see Fig. 6, and this corresponds to the movement of the CoP of the body in the plane of the platforms.

The system also allows us to monitor the contact forces (i.e. the weights) under each foot on the left or right platform and to calculate the total contact

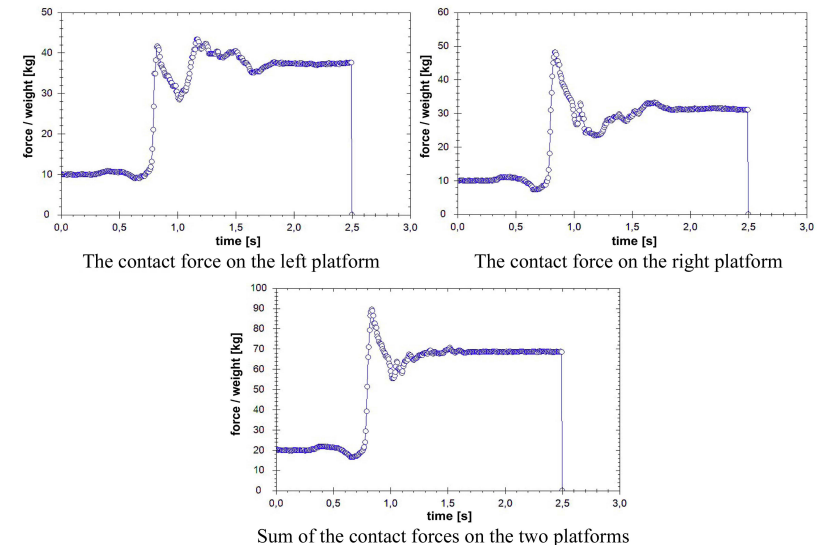

FIGURE 6. Example of the graphs of the contact forces under both feet on both platforms measured during the transition from sitting to standing.

force, i.e. the resultant contact force (i.e. weight) on the two platforms. An example of the use of the contact force measurement is shown in Fig. 6. The contact forces under both feet and the sum of the forces can also be measured during the transition from sitting to standing. The graphs of the size of the contact forces shows that both platforms were first loaded with approximately $10 \mathrm{~kg}$ (i.e. $100 \mathrm{~N}$ ), Fig. 6 . During the transition from sitting to standing, the right foot was initially more loaded than the left foot - by about $10 \mathrm{~kg}$ more, up to $50 \mathrm{~kg}$. Lower maximum values of forces during the transition were found on the left platform, but the final stable value of the contact force under the left foot after the transition from sitting to standing is higher (by about $6 \mathrm{~kg}$ ) than under the right foot. The graph of the size of the total contact forces under both feet on both platforms shows that the final stable total contact force value is approximately $700 \mathrm{~N}$, i.e. $70 \mathrm{~kg}$, which is the total body weight of the measured subject.

\section{Discussion}

As described above, our technique enables us to measure the position of $\mathrm{CoP}$ and the values of the contact forces of more complex movements. The aim was to implement a method for constructing an inexpensive new system - our new system costs less than EUR 1000. The system is based on inexpensive and widely available Wii Fit balance boards from Nintendo Ltd. The disadvantage of these inexpensive platforms is that the sampling frequency is lower than the sampling frequency of conventional platforms, e.g. the Kistler force platform. However, conventional platforms cost EUR 10000 and upwards. We have described how the relative position and orientation of platforms in 3D space are measured and evaluated, and have illustrated the functionality of our proposed technique. We used a pair of cheap gyro-accelerometer sensors to measure the relative position and orientation of the platforms. To determine the relative positions of the platforms, it is necessary to calibrate and locate the platforms in the 
initial positions in $3 \mathrm{D}$ space at the beginning of each measurement, after which no additional maintenance or system calibration is needed. In experiments with a very inexpensive gyro-accelerometer, we unfortunately discovered inaccuracies due to sensor drift, which introduced an error into the identified relative position and orientation. The solution to this problem may be to apply more precise gyro-accelerometers, which are more expensive. Alternatively, the commercial MoCap system can be used to identify the positions and orientations of the platforms in 3D space. This could be based on an optical or electromagnetic principle, but the system is much more expensive than the sensors that we have used.

\section{Conclusion}

Measurements of the CoP position and the size of the contact forces using two platforms provide a wide range of information on the measured subjects. The device that we have designed can be used as a system for medical investigations in clinical practice - podiatry, neurology [9], rehabilitation or sports medicine. Our system/ technique allows us to extend the capabilities of current platforms in clinical practice with new methods for examining a patient's posture and gait [10]. It is possible to use software that supports cognitive therapy rehabilitation through games [11], as is common in commercially available systems. It is also desirable to allow integration with other systems, such as MoCap systems for sensing the position of body segments [12], etc. A study of the position of $\mathrm{CoP}$ and contact forces using an affordable system with cheap two platforms opens up new opportunities for measurements and analyses, and also possible methods for connecting the measuring device with MoCap systems.

\section{ACKNOWLEDGEMENTS}

This work was done in the Joint Department of the Faculty of Biomedical Engineering CTU and Charles University in Prague in the framework of research program No. VG20102015002 (2010-2015, MV0/VG), sponsored by the Ministry of the Interior of the Czech Republic, and project SGS 13/091/OHK4/1T/17 CTU Prague.

\section{REFERENCES}

[1] Kriz, J., Seba, P.: Force plate monitoring of human hemodynamics. Nonlinear biomedical physics, 2(1), 2008, p. 1-9.

[2] Oatis, C.A.: Kinesiology: The Mechanics and Pathomechanics of Human Movement. Philadelphia Lippincott Williams \& Wilkins, 2009.

[3] Yelnik, A., I. Bonan, I.: Clinical tools for assessing balance disorders. Neurophysiologie Clinique (Clinical Neurophysiology), 38(6), 2008, p. 439-445.

[4] Kemeny, T., Stein, P., Havrilla, K.: Golden Book of Strain Gages, Load Cells and Brittle Coatings, Waltham: Nova Science Pub, 2008.

[5] Cho, W.H., Choi, H.: Center of Pressure (COP) During the Postural Balance Control of High-Heeled Woman. Proceedings of the IEEE Engineering in Medicine and Biology 27th Annual Conference, September 2005, p. 2761-2764.

[6] Jacobson, G.P., Newman, C.W., Kartush, J.M.: Handbook of Balance Function Testing. Andover: Cengage Learning, 1997.

[7] Landau, L.D., Lifshitz, E.M.: Mechanics, Volume 1 (Course of Theoretical Physics), Oxford: Butterworth-Heinemann, 1976.

[8] Erdos, C.G., Farkas, G., Pataki, B.: Development of a Simple and Cheap Device for Movement Analysis. IFMBE Proceedings of the XII Mediterranean Conference on Medical and Biological Engineering and Computing, 2010, 29(1), 2010, p. 61-64.

[9] Desmond, A.: Vestibular Function: Evaluation and Treatment. New York: Thieme, 2004.

[10] Sevsek, F.: Determination of sway area by Fourier analysis of its contour. Proceedings of the 6th WSEAS International Conference on Applied Computer Science (ASC'06), Tenerife, December 2006, p. 514-518.

[11] Tossavainen, T., Toppila, E., Pyykko, I., Forsman, P.M., Juhola, M., Starck, J.: Virtual reality in posturography. IEEE Transactions Information Technology in Biomedicine, 10(2), 2006, p. 282-92.

[12] Robertson, D.G.E., Caldwell, G.E., Hamill, J., Kamen, G., Whittlesey, S.N.: Research Methods in Biomechanics. Champaign: Human Kinetics, 2004. 